\title{
Posterior C2-C3 Fixation for Unstable Hangman's Fracture
}

\author{
Dong Hwan Jeong, Nam Kyu You, Chul Kyu Lee, Ki Hong Cho, Sang Hyun Kim \\ Department of Neurosurgery, Ajou University School of Medicine, Suwon, Korea
}

Objective: This is a retrospective review of 13 unstable Hangman's fractures who underwent posterior C2-3 fixation to describe clinical outcomes with a literature review.

Methods: Thirteen patients for unstable Hangman's fracture were enrolled between July 2007 and June 2010 were included in this study. The medical records of all patients were reviewed. Concurrently, clinical outcomes were evaluated using Neck Disability Index (NDI) scores and Visual Analogue Scale (VAS) scores during preoperative and postoperative follow up period. Plain radiographs were obtained on postoperative 1day, 1week, and then at 1, 2, 6, and 12 months. CT was done at postoperative 12 months in all patients for evaluation of bone fusion. The mean period of clinical follow-up was 17 months. Results: Mean age were 43 years old. Bone fusion was recognized in all cases at the final follow-up. The average preoperative VAS score for neck pain was $8.3 \pm 1.1$, while the final follow-up VAS score was $2.07 \pm 0.8(p<0.001)$. The average immediate postoperative NDI was $84 \%$ points and final NDI was $22 \%$ points $(p<0.001)$. There were one case of infection and 1 case of screw loosening.

Conclusion: In the treatment of the patients with unstable Hangman's fracture, posterior C2-C3 fusions is effective and curative treatments to achieve cervical spinal stability.

Key Words: Traumatic spondylolisthesis of the axis $\cdot$ Hangman's fracture $\cdot$ instability $\cdot \mathrm{C} 2 \cdot \mathrm{C} 3$

\section{INTRODUCTION}

Traumatic spondylolisthesis of the axis, or Hangman's fracture, is a common injury type of high cervical spine and the second most common fracture of axis. It involves bilateral C2 pars interarticularis fracture with a variable degree of displacement of $\mathrm{C} 2$ on $\mathrm{C} 3$ vertebrae ${ }^{9,19)}$. Although most Hangman's fractures are treated conservatively ${ }^{2,20)}$, surgery is usually preferable in cases of highly unstable Hangman's fracture and fusion failure after rigid arthrodesis ${ }^{3,5,14,19,21)}$. Surgical stabilization has been described using both anterior and posterior approaches $^{16,18,19,24,25)}$. The anterior approach involves a C2-C3 discectomy with interbody fusion and plating. It has the advantage of technical ease and a relative short fusion construct $^{19)}$. An anterior approach, however, does not address the detached posterior arch of $\mathrm{C} 2$ and instability remained during flexion and extension ${ }^{7}$. Among the different posterior approaches, direct repair of the pars fracture with a screw across the fracture

- Received: August 17, 2013 - Revised: September 3, 2013

- Accepted: September 5, 2013

Corresponding Author: Sang Hyun Kim, MD

Department of Neurosurgery, Ajou University School of Medicine, Suwon 443-721, Korea

Tel: +82-31-219-5230/5235, Fax: +82-31-219-5238

E-mail: shkim709@ajou.ac.kr line has the advantage of preserving motion of the axis ${ }^{3,5,13,211}$. Unfortunately, direct pars repair does not address instability at the disc. Although posterior C1-C3 wiring techniques have also been described, these techniques have required postoperative halo-thoracic immobilization ${ }^{14)}$. Pos-terior C1-C3 screw and rod fixation can offer good results in terms of alignment and stabilization of spine with a low level of pseudoarthrosis $^{12}$. This technique, however, can not preserve the motion at intact C1-C2 segment. Another posterior stabilization strategy uses a $\mathrm{C} 2$ pars screw connected to a $\mathrm{C} 3$ lateral mass screw. This technique addresses the detached posterior arch of $\mathrm{C} 2$ by pinning the fractured pars while simultaneously addressing instability at the disc by immobilizing $\mathrm{C} 2$ relative to $\mathrm{C} 3$. Therefore, posterior $\mathrm{C} 2-\mathrm{C} 3$ fixation more effectively stabilizes a hangman's fracture than anterior cervical plating ${ }^{7}$.

In this study, we review our clinical experience of posterior C2-C3 fixation for the 13 patients with unstable Hangman's fracture and evaluated the clinical and radiological outcomes with a literature review.

\section{PATIENTS AND METHODS}

Thirteen consecutive patients with unstable Hangman's fracture were enrolled who required posterior C2-C3 fixation using the Polyaxial Screw-rod System (DePuy/Synapse, Switzer- 
land) between July 2007 and June 2010. The medical records of all patients were reviewed. All patients were classified and selected using Classification of hangman's fracture which was proposed by Levine and Edwards to evaluate ${ }^{15,16}$. Type I has stable and minimal translation $(<3 \mathrm{~mm})$ without $\mathrm{C} 2-\mathrm{C} 3$ angulation. Type II has unstable disc disruption and significant C2$\mathrm{C} 3$ angulation and translation ( $>3 \mathrm{~mm}$ ). Type IIA is unstable due to flexion-distraction injury and has more angulation than type 2 without translation (Fig. 1A, B). Type III is unstable and has severe C2-C3 angulation and translation (Fig. 1C). It has sometimes unilateral or bilateral facet disloca- tion. Surgical stabilization was recommended in Levine-Edwards Type IIa and III fractures with significant unstable dislocation. Surgical treatments were performed in patient who showed poor reduction or nonunion after rigid external orthosis, neurologic deficit or unstable combination fracture at the presentation.

Dynamic plain radiographs were obtained on postoperative 1 day, 1 week, and then at 1, 2, 6, and 12 months and Computed Tomographic (CT) evaluation was done at postopera- tive 12 months in all patients for evaluation of bone fusion. The adequacy of fusion was determined at approximately four, six and 12 months postoperatively. Pseudoarthrosis was defined as a motion $>2.0 \mathrm{~mm}$ between the laminae at the base of the spinous processes between the upper and lowermost fixed and fused levels on the flexion/extension plain radiographs.

Concurrently, clinical outcomes were evaluated using Neck Disability Index (NDI) scores and Visual Analogue Scale (VAS) scores during preoperative and postoperative follow up peri$\operatorname{od}^{22)}$.

The measurement was assessed using a 10-point VAS with endpoint anchors of no pain (0 points) and severe pain (10 points). The NDI questionnaire is comprised of 10 single items related to activities of daily living. Each item has six predefined response categories, coded $0-5$ on an ordinal scale. The scores reflected either the degree of neck pain or the degree of difficulty in performing certain actions due to neck pain. The lowest score (0) represented no problem or pain, whereas the highest score (5) represented maximum problems or pain. The pre- and postoperative arm and neck symptom VAS scores and NDI scores were compared using two-sample $t$ tests paired for means. A P value of $<0.05$ was regarded as significant

\section{Surgical Technique}

The surgery was performed in a consistent fashion. The patient was carefully turned to the prone position and the patient's head was placed in a Mayfield Cranial Fixation device with fluoroscopic guidance to assess the spinal alignment
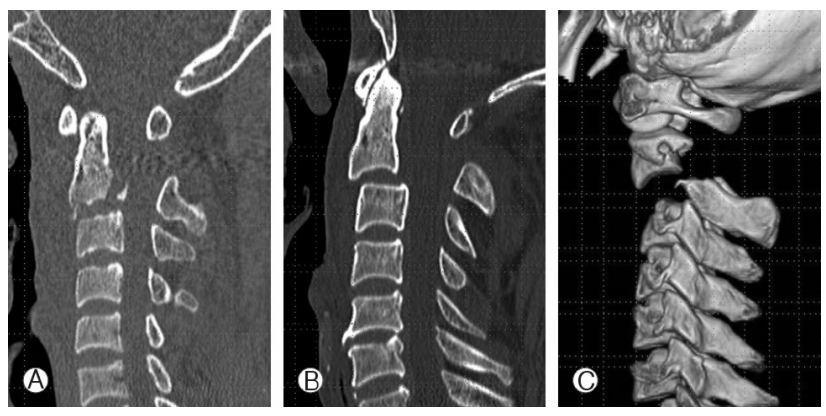

Fig. 1. Computed tomographic images for Type lla (A, B) and Type III (C) Hangman's fracture.
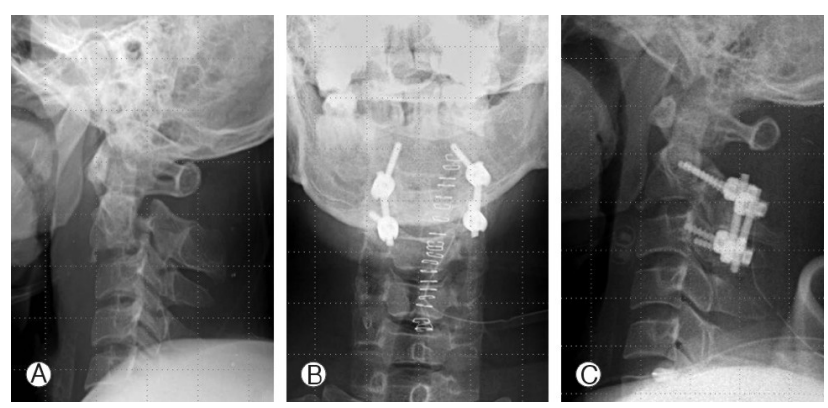

Fig. 2. Preoperative cervical spine lateral view (A) of Type lla Hangman's fracture, postoperative anteroposterior (B) and lateral (C) views. Pedicle screws were used at $\mathrm{C} 2$ and lateral mass screws were used at C3 under the C-arm guidance.

as well as to localize the C2-C3 levels. The patient could then be prepped and draped in the standard fashion.

A standard midline incision was made above the C1-C4 levels. The lateral masses were exposed in a subperiosteal fashion to the lateral margins of the facet joints. Once the deformity was reduced, the lateral masses to be fused were decorticated with the drill. Great care was taken not to disturb the capsule at adjacent levels in order to prevent any iatrogenic instability. Pedicle screws were used at $\mathrm{C} 2$ and lateral mass screws were used at $\mathrm{C} 3$ under the $\mathrm{C}$-arm guidance (Fig. 2). A rod was prepared to the appropriate length and contour that it would easily pass through the heads of all polyaxial screws. Once the rod was positioned, it was secured to the heads of the screws using outer nuts.

Bony fusion was performed by packing allograft bone and demineralized bone matrix (DBM) into the facet joints, around the decorticated lateral masses lateral to the rod and laminae. During the entire procedure, the retractors were intermittently released to avoid denervation of the erector spinal muscles. Routine closure was carried out and drains were left in place as needed. 


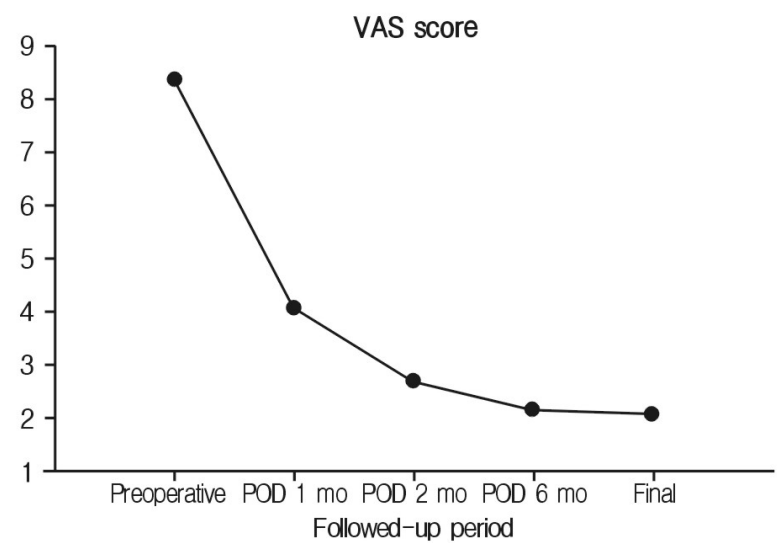

Fig. 3. The average preoperative VAS score for neck pain was $8.3 \pm 1.1$. VAS score was gradually decreased during the followup period and the mean VAS score was decreased up to 2.07士 0.8 at final follow-up. This difference between preoperative and final follow-up VAS score was statistically significant $(p<0.001)$ by paired T-test.

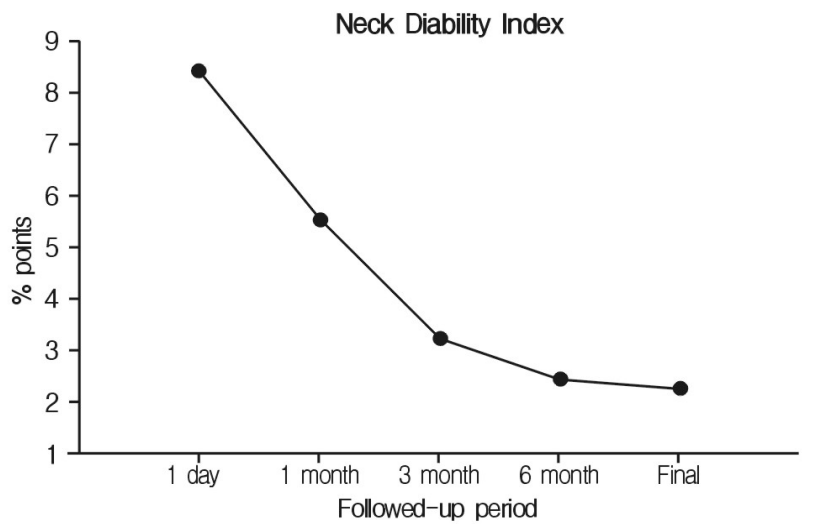

Fig. 4. The average immediate postoperative NDI was $84 \%$ points. $\mathrm{NDI}$ was gradually decreased during the follow-up period and the mean NDI was decreased up to $22 \%$ points at final follow-up. This difference between preoperative and final follow-up NDI score was statistically significant $(p<0.001)$ by paired T-test.

\section{RESULTS}

Five patients with Type IIa Hangman's fracture and 8 patients with Type III Hangman's fracture were enrolled. The patients included 8 men and 5 women. Mean age was 43 years old (range 18 to 64 years). The mean follow-up period was 17.2 months (range 12 to 32 months).

The average preoperative VAS score for neck pain was $8.3 \pm 1.1$. VAS score was gradually decreased during the follow-up period and the mean VAS score was decreased up to $2.07 \pm 0.8$ at final follow-up. This difference between preoperative and final follow-up VAS score was statistically sig-

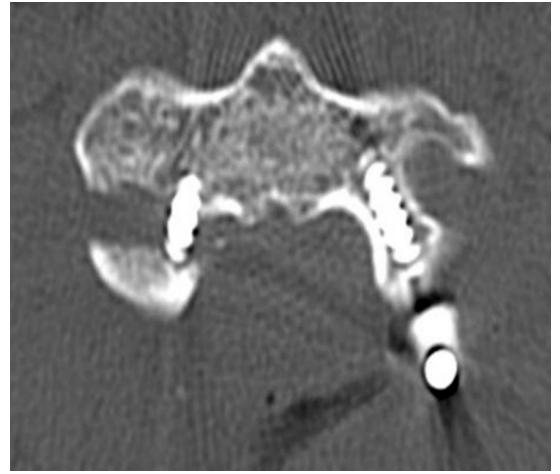

Fig. 5. Immediate postoperative CT scan was performed in the patient with screw malposition which showed suboptimal screw placement with wide fracture gap.

nificant $(\mathrm{p}<0.001)$ by paired T-test (Fig. 3).

The average immediate postoperative NDI was $84 \%$ points. NDI was gradually decreased during the follow-up period and the mean NDI was decreased up to $22 \%$ points at final follow-up. This difference between preoperative and final follow-up NDI score was statistically significant $(\mathrm{p}<0.001)$ by paired T-test (Fig. 4).

There were one case of wound infection and 1 case of screw malposition. Immediate postoperative CT scan was performed in the patient with screw malposition which showed suboptimal screw placement with wide fracture gap (Fig. 5). Revision surgery for screw repositioning was needed in case of screw loosening. Solid Bone fusion was confirmed in all cases at the final follow-up.

\section{DISCUSSION}

Hangman's fracture is the second most common fracture of the second cervical vertebra (C2). It involves a bilateral arch fracture of the $\mathrm{C} 2$ pars interarticularis with variable displacement of $\mathrm{C} 2$ on $\mathrm{C}^{7}$. Among different classifications for Hangman's fracture, Levine-Edward's is the most frequently used $^{9-11,15)}$. Type I lesion is usually considered stable with no angulation and displacement on C2-C3, whereas Types II, IIa, and III are usually considered unstable. These unstable types are combined with the injury of the $\mathrm{C} 2-\mathrm{C} 3$ disc and the anterior longitudinal ligament. In Type II and type III Hangman's fracture, extension forces have been implicated in the disruption of the anterior longitudinal ligament, posterior longitudinal ligament, and $\mathrm{C} 2-\mathrm{C} 3$ disc.

In most cases of Hangman's fracture, the conservative treatment is the most indicated ${ }^{4,6,10,20,21,23)}$. Semi-rigid collars are used for cases with a small degree of dislocation. Rigid immobilizations such as halo-vest are used for cases of great 
displacement. In regard to the unstable Hangman's fracture, the treatment is still controversial. Pseudoarthrosis, anterior dislocation, angulation of $\mathrm{C} 2$ over $\mathrm{C} 3$, and recurrent axial pain were observed in about $60 \%$ of the cases of Types II, IIa, and III, which were primarily treated with conservative therapy ${ }^{10,11,20)}$. Majority of authors recently suggest that the presence of discoligamentous injury on top of bony injury would require surgery. Another reason for surgery is the shortening the course of treatment ${ }^{3-4,19,21,24-25)}$.

The goals in the surgical treatment of Types II and III Hangman's fracture are reduction, stabilization, and maintenance of alignment. Surgical stabilization has been described in both anterior and posterior approaches. The anterior approach, which has the advantage of technical ease and a relatively short fusion construct, involves a C2-C3 discectomy with interbody fusion and plating ${ }^{16,25)}$. An anterior approach, however, does not address the detached posterior arch of C2, and the anterior interbody fusion of $\mathrm{C} 2-\mathrm{C} 3$ alone is inefficient for 3 column discoligamentous injuries like Types II and III Hangman's fracture. Among the different posterior approaches, direct repair of the pars fracture with a screw across the fracture line has the advantage of preserving motion of the axis ${ }^{3,5,16}$.

However, direct pars repair does not address instability at the disc. The main advantage of this technique is not having to sacrifice any normal motion of the $\mathrm{C} 2$ segment and fixating only the fractured bones of $\mathrm{C} 2$. However, it can be used only in cases with minimal or no $\mathrm{C} 2-\mathrm{C} 3$ disc injury. It is ineffective for instability at the C2-C3 level. Although posterior C1-C3 wiring techniques have also been described, these techniques require postoperative halo-thoracic immobilization ${ }^{4)}$.

Polyaxial screw and rod fixation system has been developed and is widely used as a standard instrument. In this system, the screws can be placed first and the rod can be contoured in multiple planes. As a result, the screw?rod system is able to more effectively accommodate variations in size, spacing, and morphology of the lateral masses. Recent studies have also reported that the biomechanical comparison of stabilization techniques on Hangman's fracture and posterior C2-C3 screw and rod construction was found to be more effective on the stabilization of Hangman's fracture than anterior cervical plating and $\mathrm{C} 2$ pars screwing ${ }^{7}$. In the same cadaver study, this technique provided significantly better biomechanical stability, especially during lateral bending and axial rotation compared with anterior $\mathrm{C} 2-\mathrm{C} 3$ plating. Furthermore, screw fixation of $\mathrm{C} 2-\mathrm{C} 3$ has 3 advantages. First, it provides $\mathrm{C} 2-\mathrm{C} 3$ stabilization and restoration of the posterior elements of the axis. Second, the construct behaves similarly to the tension bands against flexion, lateral bending, and axial rotation ${ }^{2,7}$. Third, there is no need for a halo vest immobilization after surgery. Pedicle screw fixation is a biomechanically stronger repair method and requires only a posterior approach ${ }^{1,17}$. In addition, pedicle screw instrumentation through a single stage posterior approach offered better biomechanical stability in 3 column spinal injury. However, pedicle screw placement is technically demanding because of large individual variations in the pedicle dimensions and course of the vertebral artery.

Therefore, the successful placement of cervical pedicle screws requires a 3-dimensional knowledge of the pedicle morphology to identify ideal screw axis accurately and to avoid neurovascular injury ${ }^{1,8,17}$. CT scanning with 3-dimensional reconstruction or an Magnetic Resonance Image (MRI) evaluation of the spine is essential for the detection of individual variations in the dimensions of the pedicle before surgery.

Although posterior C2-C3 fixation for unstable Hangman's fracture offers biomechanical stability without additional treatment, there are some prerequisite conditions for achievement of good clinical and radiological results. Complete reduction and close adhesion of fracture gap should be achieved before the insertion of $\mathrm{C} 2$ screw. And, we have to press down the screw during insertion of $\mathrm{C} 2$ pedicular screw not to make fracture gap wide. Even if complete reduction is not achieved before the insertion of pedicle screws, bicortical purchase of $\mathrm{C} 2$ pedicular screw, or posterior C1-C3 fixation, is recommended.

\section{CONCLUSION}

In the treatment of the patients with unstable Hangman's fracture, posterior $\mathrm{C} 2-\mathrm{C} 3$ fixation is an effective and curative treatments to achieve cervical spinal stability. Posterior C2-C3 fixation can offer high solid fusion rate and good clinical outcomes for axial neck pain. Preservation of motion can be achieved because of short segment fusion at index levels. Although further studies are required to compare its clinical results with other posterior fixation, we cautiously suggest that the posterior $\mathrm{C} 2$ to $\mathrm{C} 3$ fusion can be considered for primary surgical treatment in patient with unstable Hangman's fracture when we can achieve complete reduction intraoperatively.

\section{REFERENCES}

1. Abumi, Shono, Ito, Taneichi, Kotani, Kaneda: Complications of pedicle screw fixation in reconstructive surgery of the cervical spine. Spine (Phila Pa 1976) 25:962-969, 2000

2. Arand, Neller, Kinzl, Claes, Wilke: The traumatic spondylolisthesis of the axis. A biomechanical in vitro evaluation of an instability model and clinical relevant constructs for stabilization. Clin Biomech (Bristol, Avon) 17:432-438, 2002

3. Borne, Bedou, Pinaudeau: Treatment of pedicular fractures of the axis. A clinical study and screw fixation technique. J Neurosurg 60:88-93, 1984 
4. Bridwell: Treatment of a markedly displaced hangman's fracture with a luque rectangle and a posterior fusion in a 71-year-old man. Case report. Spine (Phila Pa 1976) 11:49-52, 1986

5. Bristol, Henn, Dickman: Pars screw fixation of a hangman's fracture: technical case report. Neurosurgery 56:E204; discussion E204, 2005

6. Coric, Wilson, Kelly: Treatment of traumatic spondylolisthesis of the axis with nonrigid immobilization: a review of 64 cases. J Neurosurg 85:550-554, 1996

7. Duggal, Chamberlain, Perez-Garza, Espinoza-Larios, Sonntag, Crawford: Hangman's fracture: a biomechanical comparison of stabilization techniques. Spine (Phila Pa 1976) 32:182-187, 2007

8. Ebraheim, Rollins, Xu, Jackson: Anatomic consideration of C2 pedicle screw placement. Spine (Phila Pa 1976) 21:691-695, 1996

9. Effendi, Roy, Cornish, Dussault, Laurin: Fractures of the ring of the axis. A classification based on the analysis of 131 cases. J Bone Joint Surg Br 63-B:319-327, 1981

10. Fielding, Francis, Hawkins, Pepin, Hensinger: Traumatic spondylolisthesis of the axis. Clin Orthop Relat Res:47-52, 1989.

11. Francis, Fielding: Traumatic spondylolisthesis of the axis. Orthop Clin North Am 9:1011-1027, 1978

12. Hadley, Browner, Sonntag: Axis fractures: a comprehensive review of management and treatment in 107 cases. Neurosurgery 17:281-290, 1985

13. HJ Choi, KS Kim, KS Park, IH Han, DK Chin, BH Jin, et al: Technical strategies and surgical results of C1 lateral mass - C2 pedicular screw fixation in atlatoaxial disorders. Kor J Spine 5(3):196-202, 2008

14. Karimi-Nejad: Surgical management of cervical spine injuries. Neurosurg Rev 12 Suppl 1:525-535, 1989
15. Levine, Edwards: The management of traumatic spondylolisthesis of the axis. J Bone Joint Surg Am 67:217-226, 1985

16. Li, Dai, Lu, Chen: A systematic review of the management of hangman's fractures. Eur Spine J 15:257-269, 2006

17. Ludwig, Kowalski, Edwards, Heller: Cervical pedicle screws: comparative accuracy of two insertion techniques. Spine (Phila Pa 1976) 25:2675-2681, 2000

18. Muller, Wick, Muhr: Traumatic spondylolisthesis of the axis: treatment rationale based on the stability of the different fracture types. Eur Spine J 9:123-128, 2000

19. Tuite, Papadopoulos, Sonntag: Caspar plate fixation for the treatment of complex hangman's fractures. Neurosurgery 30: 761-764; discussion 764-765, 1992

20. Vaccaro, Madigan, Bauerle, Blescia, Cotler: Early halo immobilization of displaced traumatic spondylolisthesis of the axis. Spine (Phila Pa 1976) 27:2229-2233, 2002

21. Verheggen, Jansen: Hangman's fracture: arguments in favor of surgical therapy for type II and III according to Edwards and Levine. Surg Neurol 49:253-261; discussion 261-252, 1998

22. Vernon, Mior: The Neck Disability Index: a study of reliability and validity. J Manipulative Physiol Ther 14:409-415, 1991

23. Wilson: Treatment of traumatic spondylolisthesis of the axis with nonrigid immobilization: erratum. J Neurosurg 85:1198, 1996

24. Wilson, Marshall, Ewart: Transoral fusion with internal fixation in a displaced hangman's fracture. Spine (Phila $\mathrm{Pa} 1976) 24$ : 295-298, 1999

25. Ying, Wen, Xinwei, Yong, Hongyu, Zhu, et al: Anterior cervical discectomy and fusion for unstable traumatic spondylolisthesis of the axis. Spine (Phila Pa 1976) 33:255-258, 2008 